\title{
Verification of the compound nuclear model for T-violation search
}

T. Yamamoto*, H.M.Shimizu, K.Ishizaki, Y.Niinomi, M.Kitaguchi' ${ }^{2}$, S.Takada ${ }^{3}$, J.Koga $^{3}$, S.Makise ${ }^{3}$, T.Yoshioka ${ }^{4}$, A.Kimura ${ }^{5}$, K.Sakai $^{5}$, T.Oku ${ }^{5}$, T.Okudaira ${ }^{5}$,

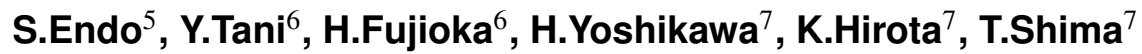

Nagoya University

${ }^{2}$ Nagoya University KMI

${ }^{3}$ Kyиsуu University

${ }^{4}$ Kyusyu University RCAPP

${ }^{5} J A E A$

${ }^{6}$ Tokyo Institute of Technology

${ }^{7}$ Osaka University RCNP

E-mail: yamamoto@phi.phys.nagoya-u.ac.jp

Large sources of parity violation have been observed in compound nuclear reactions. These enhancements are described by the compound nuclear model, which can be used to describe the angular correlation of $\gamma$-rays produced in $(n, \gamma)$ reactions in medium-heavy nuclei. It has also been suggested that these same reactions could lead to a similar enhancement in time reversal violating processes. Therefore, we are interested in verifying the compound nuclear model by studying the $(\mathrm{n}, \gamma)$ reaction using polarized neutrons. In this paper, we discuss the installation and characterization of a neutron polarizer used in the $(n, \gamma)$ measurement with transversely polarized neutrons.

The 4th KMI International Symposium (KMI2019)

18-20, February 2019

Nagoya, Japan

${ }^{*}$ Speaker. 


\section{Introduction}

The current matter-dominated universe needs CP-violation (and by the CPT Theorem, a violation of CP implies T-violation) in order to explain the observed matter-antimatter asymmetry. However the known T-violation confirmed by experiments is too small to explain the discrepancy. This fact strongly suggests the existence of additional (unknown) sources of T-violation. We are searching for T-violation in nuclear reactions.

Large parity violating effects have been observed in compound nuclear reactions between mediumheavy nuclei and neutrons. It is understood that this large symmetry violation is a result of an amplification of symmetry breaking in elementary weak interaction processes. The compound nuclear model (CNM also predicts that the same amplification mechanism could enhance possible T-violating processes in this system [1]. Therefore, we wish to verify the compound nuclear reaction theory in detail and evaluate the feasibility of a T-violation search in such nuclear reaction processes. CNM predicts angular correlations in $(n, \gamma)$ reaction in the following formula [2]:

$$
\begin{aligned}
\frac{d \sigma_{n \gamma}}{d \Omega_{\gamma}}= & \frac{1}{2}\left\{a_{0}+a_{1} \hat{k}_{n} \cdot \hat{k}_{\gamma}+a_{2} \sigma_{n} \cdot\left(\hat{k}_{n} \times \hat{k}_{\gamma}\right)+a_{3}\left(\left(\hat{k}_{n} \cdot \hat{k}_{\gamma}\right)^{2}-\frac{1}{3}\right)\right. \\
& +a_{4}\left(\hat{k}_{n} \cdot \hat{k}_{\gamma}\right)\left(\sigma_{n} \cdot\left(\hat{k}_{n} \times \hat{k}_{\gamma}\right)\right)+a_{9} \sigma_{n} \cdot \hat{k}_{\gamma}+a_{10} \sigma_{n} \cdot \hat{k}_{n} \\
& \left.+a_{11}\left(\left(\sigma_{n} \cdot \hat{k}_{\gamma}\right)\left(\hat{k}_{\gamma} \cdot \hat{k}_{n}\right)-\frac{1}{3}\left(\sigma_{n} \cdot \hat{k}_{n}\right)\right)+a_{12}\left(\sigma_{n} \cdot \hat{k}_{n}\right)\left(\left(\hat{k}_{n} \cdot \hat{k}_{\gamma}\right)-\frac{1}{3}\left(\sigma_{n} \cdot \hat{k}_{\gamma}\right)\right)\right\}(1.1)
\end{aligned}
$$

where $\hat{k}_{n}, \hat{k}_{\gamma}, \sigma_{n}$, are unit vectors parallel to the neutron momentum, $\gamma$-ray momentum, and neutron spin, respectively, and $a_{0}, a_{1}, . ., a_{12}$ are correlation coefficients describing the angular- and polarization- dependent contributions to the resonance parameters. The $a_{2}, a_{9}, a_{10}, a_{11}$, and $a_{12}$ terms depend on the neutron polarization. The angular correlation in the ${ }^{139} \mathrm{La}(\mathrm{n}, \gamma)$ reaction using unpolarized neutrons was previously measured by Okudaira et al, and the results support the CNM [3]. However these measurements were not sensitive to the aforementioned terms that depend on the neutron polarization. There has not yet been a measurement of the angular distribution of the ${ }^{139} \mathrm{La}(\mathrm{n}, \gamma)$ reaction using polarized neutrons. Therefore, designing an experiment that will allow for such a measurement is of interest. We used a ${ }^{3} \mathrm{He}$ spin filter as a neutron polarizer. We installed this neutron polarizer onto beamline BL04 of the Material and Life Science Experimental Facility (MLF) of the Japan Proton Accelerator Research Complex (J-PARC) and measured the $a_{2}$ term using polarized neutrons for the first time.

\section{Experiment}

The setup at BL04 is shown in Figure 1. A solenoid coil used to produce a magnetic field to maintain the ${ }^{3} \mathrm{He}$ polarization was installed perpendicular to the beam axis. In the experiment, the ${ }^{3} \mathrm{He}$ glass cell was polarized off of the beamline and then reinstalled onto the beamline. The ${ }^{139} \mathrm{La}$ target was irradiated with polarized neutrons and the emitted $\gamma$-rays were measured by array of Ge detectors. The neutrons transmitted through the target were measured by a downstream transmission neutron detector. The polarization direction of the neutron was controlled by flipping the spin of the ${ }^{3} \mathrm{He}$ using the adiabatic fast passage method (AFP-NMR). Furthermore, the polarization 
ratio of ${ }^{3} \mathrm{He}$ was monitored using a NMR signal. The solenoid is $30 \mathrm{~cm}$ in diameter and $40 \mathrm{~cm}$ in length, with a magnetic field strength of around 15 Gauss at the center. The ${ }^{3} \mathrm{He}$ cell was $7 \mathrm{~cm}$ in length and had a gas pressure of $3 \mathrm{~atm}$.

Ge detector array

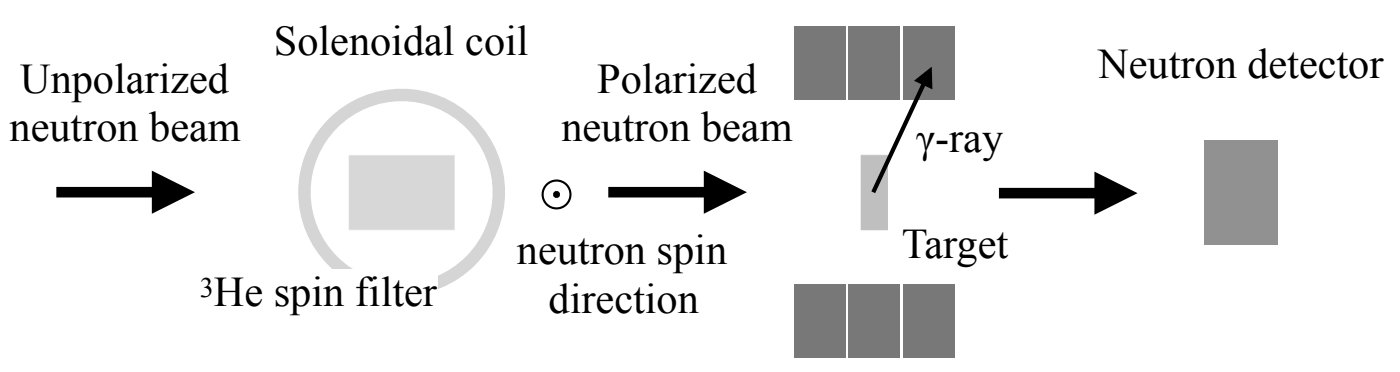

Figure 1: A side view of the experimental setup at BL04.

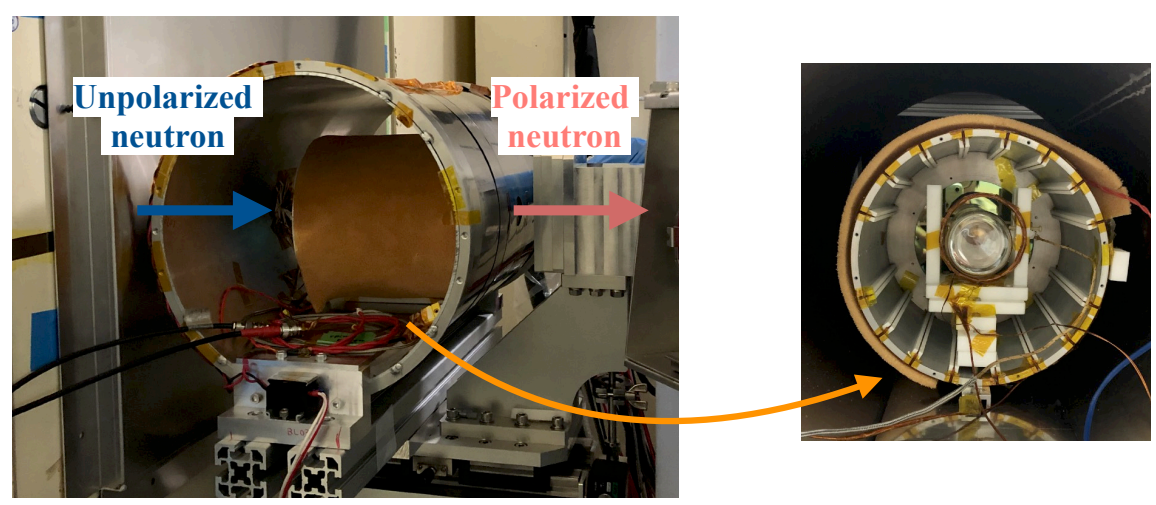

Figure 2: The figure on the left is a photograph of the ${ }^{3} \mathrm{He}$ spin filter installed onto the beamline, and the one on the right is a photograph of the ${ }^{3} \mathrm{He}$ cell and NMR apparatus.

This measurement took approximately 32 hours in total. The direction of the neutron helicity was changed between positive and negative states every few hours. The neutron polarization ratio was analyzed using the transmittance of the ${ }^{3} \mathrm{He}$ spin filter. Figure 3 shows the time dependence of the polarization ratio of the ${ }^{3} \mathrm{He}$. After the installation of the ${ }^{3} \mathrm{He}$ spin filter, we could see that the polarization relaxed with time. We estimated that the polarization relaxation time of the ${ }^{3} \mathrm{He}$ was $(127.9 \pm 0.3) \mathrm{h}$. The average neutron polarization ratio was approximately $15 \%$. This result indicates that the relaxation time of the ${ }^{3} \mathrm{He}$ on the beamline is sufficiently slow enough to be able to measure $a_{2}$. Therefore we plan to measure $a_{2}$ with sufficient statistics (3-4 days) and higher ${ }^{3} \mathrm{He}$ polarization during the next beamtime.

\section{Summary}

We want to search for T-violation in nuclear reactions. In order to do so, we must first verify 


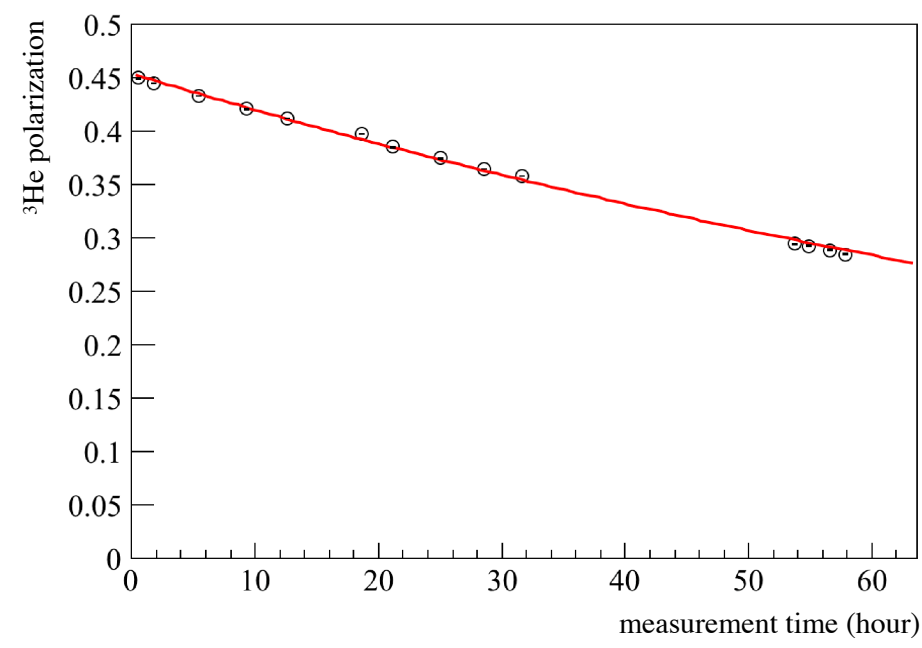

Figure 3: Fig. 3 shows the ${ }^{3} \mathrm{He}$ polarization relaxation as a function of time.

the CNM . We wish to measure the $a_{2}$ correlation term, which requires a polarized neutron beam. Therefore, it is necessary to install a ${ }^{3} \mathrm{He}$ polarizer onto the neutron beam line. Since we it was found that the polarizer has a long relaxation time, we plan to measure $a_{2}$ with high statistical precision. $\mathrm{CN}$

\section{Acknowledgement}

The neutron experiment at the Materials and Life Science Experiment Facility of the J-PARC was performed under a user program (Proposal No. 2018B0148). This work was supported by the Neutron Science Division of KEK as S- type research projects with program numbers 2014S03 and 2018S12. This work was partially supported by MEXT KAKENHI Grant No. JP19GS0210 and JSPS KAKENHI Grant No. JP17H02889.

\section{References}

[1] V. P. Gudkov, Phys. Rep. 212, 77 (1992)

[2] V. V. Flambaum et al., Nucl. Phys. A 13412 (1984)

[3] T. Okudaira et al., Phys. Rev. C 97034622 (2018) 